\title{
Efectos de la poda de tallo y fertilización de liberación controlada en vivero sobre el comportamiento de plántulas de Nothofagus nervosa en terreno
}

\author{
Effects of top pruning and slow-release fertilization in the nursery on the performance \\ of outplanted Nothofagus nervosa seedlings
}

\author{
Pablo J Donoso ${ }^{a}$, Daniel P Soto ${ }^{\text {a*, Víctor Gerding }}{ }^{\mathrm{a}}$ \\ *Autor de correspondencia: aniversidad Austral de Chile, Facultad de Ciencias Forestales, Instituto de Silvicultura, \\ casilla 567, Valdivia, Chile, tel.: 56-63-221742, fax: 56-63-221230, danielsoto@uach.cl
}

\begin{abstract}
SUMMARY
Raulí (Nothofagus nervosa) is a highly valuable and fast growing Chilean native tree species, therefore being of great interest for the establishment of plantations. The success of plantations depends upon several factors, among which nursery management can be decisive. This study evaluated the effects of slow release fertilization (FLC) in doses of $2.5,5.0$ and $7.5 \mathrm{~kg} \mathrm{~m}^{-3}$, and of topping (with and without) on the survival and growth of rauli in a plantation with restoration purposes in the Valdivian Andes of Chile (650 $\mathrm{m}$ asl). Average survival was $82 \%$ the first year, $70 \%$ the second and $62 \%$ the third (mortality due to frosts). The mean height growth for the period was $120 \mathrm{~cm}$. However, the trend was that survival and growth were greater with the two higher fertilization doses, and to a lesser extent with topping. During the first three years of field evaluation we did not detect significant differences of FLC and top pruning on survival, height growth and diameter at breast height among the six treatments. However, the trend was that there were higher survival rates and growth rates with the two higher FLC doses, which also occurred with top pruning (better perforrmance with pruning), but to a lower extent. Results show that with the range of plant quality used, similar performance results can be obtained when outplanted in the field.
\end{abstract}

Key words: restoration, abiotic stress, mortality, growth.

\section{RESUMEN}

Nothofagus nervosa (raulí) es una de las especies nativas chilenas de mayor valor y crecimiento y, por ello, en el último tiempo concita alto interés para el establecimiento de plantaciones. El éxito del establecimiento de plantaciones depende de diversos factores, entre los cuales el manejo en vivero puede ser decisivo. El presente estudio evaluó los efectos de fertilización de liberación controlada (FLC en dosis de 2,5, 5,0 y 7,5 $\mathrm{kg} \mathrm{m}^{-3}$ ) y de poda apical (con y sin) en vivero sobre la supervivencia y el crecimiento de $N$. nervosa en plantaciones con fines de restauración en Los Andes valdivianos de Chile (650 m s.n.m.). La supervivencia fue de $82 \%$ en el primer año, $70 \%$ en el segundo y $62 \%$ en el tercero (mortalidad por heladas). El crecimiento promedio en altura durante el trienio estuvo alrededor de $120 \mathrm{~cm}$. Durante los primeros tres años de evaluación en terreno no se observaron efectos significativos de las dosis de FLC y la poda sobre la supervivencia, crecimientos en altura y en diámetro a la altura del pecho. Sin embargo, la tendencia fue de mayor supervivencia y crecimiento con ambas dosis mayores de FLC, lo cual también ocurrió con poda apical, pero en menor magnitud. Los resultados demuestran que en el rango de calidad de planta obtenida en vivero se pueden alcanzar similares resultados en terreno.

Palabras clave: restauración, estrés abiótico, mortalidad, crecimiento.

\section{INTRODUCCIÓN}

Los bosques degradados del sur de Chile suman más de dos millones de hectáreas entre las regiones del Bíobío y de Los Lagos $\left(38-42^{\circ} \mathrm{S}\right)$, constituyendo una oportunidad para restaurar las bases ecológicas y productivas de estos ecosistemas a través de plantaciones con especies nativas. Bajo este escenario, la producción de plantas de calidad contribuiría al éxito del establecimiento y al buen crecimiento inicial de la repoblación (Duryea y Landis 1984, Duryea y Dougherty 1991, Donoso et al. 1999).
Extensas superficies de Los Andes valdivianos han sido sometidas a explotación forestal (Otero 2006) con la finalidad principal de obtener madera de Nothofagus nervosa (Phil.) Dim. et Mil. (raulî́), una de las especies nativas chilenas más cotizadas por su alta calidad y el alto valor de su madera (Hall y Witte 1998, Díaz-Vaz et al. 2002). Como consecuencia, muchas áreas antiguamente cubiertas por densos bosques hoy constituyen zonas degradadas ocupadas principalmente por una densa cobertura de Chusquea spp., impidiendo la regeneración de ciertas especies arbóreas (González et al. 2002). En estos sitios, así como en otros con condiciones 
restrictivas (suelos degradados y climas adversos) se requiere plantar con plantas de alta calidad, es decir, que permitan altas tasas de supervivencia y de crecimiento (Wilson y Jacobs 2006), y que sean útiles para restaurar la diversidad del sistema a través de la facilitación para el ingreso de otras especies. Por ello el acondicionamiento de plantas en vivero juega un rol relevante (Duryea y Dougherty 1991, Holmes y Richardson 1999). Plantas producidas en contenedor y con poda apical son más resistentes al desecamiento, heladas, estrés hídrico y al daño por exposición de raíces que las plantas a raíz desnuda y de gran desarrollo en altura (Day et al. 2006, Donoso et al. 2007b). Por ello, las plantas producidas en contenedor, si alcanzan un adecuado estándar de calidad, presentan posteriormente en terreno menor mortalidad y mayor crecimiento (Duryea y Dougherty 1991, Wilson y Jacobs 2006).

Tradicionalmente, la fertilización junto con el riego son las actividades culturales de mayor efecto en vivero, influenciando la calidad de plantas para la repoblación (Duryea y Landis 1984). La fertilización puede provocar un aumento o un menoscabo en el crecimiento de las plantas, tanto en la parte aérea como radicular, alterando el nivel nutricional de los tejidos y su consecuente reserva. Por consiguiente, esta práctica de vivero puede afectar la capacidad de arraigamiento, la resistencia al estrés hídrico y térmico y la susceptibilidad a enfermedades (Oliet $e t$ al. 1999, Alzugaray et al. 2004, Fan et al. 2004, Rose et al. 2004, Jacobs et al. 2005). Los esquemas de fertilización en vivero han cambiado en los últimos 20 años y, actualmente, la incorporación de fertilizantes de entrega controlada (FLC) en el tiempo ha sido ampliamente utilizada y validada por permitir mejores tasas de supervivencia y crecimientos en comparación a la fertilización tradicional (Oliet et al. 1999, Rose et al. 2004, Jacobs et al. 2005). La aplicación de FLC en vivero se hace con la finalidad de nutrir las plantas durante su desarrollo en esta etapa y, si la dosis es apropiada, también puede servir de fertilizante durante los primeros años de crecimiento en la plantación en terreno. En el caso de fertilización con Osmocote, Hathaway y Whitcomb (1977) encontraron que plantas en contenedor con mayores dosis de fertilizante tuvieron mejor desarrollo posterior una vez plantadas en terreno.

Adicionalmente hay una serie de atributos morfológicos de las plantas que en general se relacionan con la capacidad de supervivencia posterior en las plantaciones. Entre éstos generalmente han sido buenos predictores la altura y peso seco de la parte aérea, el diámetro de cuello de la raíz, el peso seco radical (Navarro et al. 2006, Wilson y Jacobs 2006). De hecho, la altura en general es la variable más citada como buena predictora del desarrollo de plantas en terreno (Wilson y Jacobs 2006). En ambientes mediterráneos Navarro et al. (2006) señalan que a pesar de haber variaciones en los resultados, en general hay una relación positiva entre altura de plantas y el desarrollo de éstas. Sin embargo, en ambientes templados lo reportado por Wilson y Jacobs (2006) sugiere lo contrario, es decir que a mayor altura de planta es menor la supervivencia. La variable altura de planta puede controlarse a través del desarrollo de distintos protocolos de producción de planta (Bustos et al. 2008) o por medio de la poda apical antes de enviar las plantas a terreno.

El presente estudio plantea como objetivo evaluar el efecto de prácticas de manejo en vivero sobre la supervivencia y el crecimiento en los primeros tres años de plantación. Específicamente se considera el manejo nutritivo en vivero de distintas dosis de FLC y la aplicación de poda apical (topping) en las plantas antes de ser llevadas a terreno para plantación.

\section{MÉTODOS}

El estudio se localizó en la Cordillera de Los Andes de Valdivia, Chile, a una altitud de $650 \mathrm{~m}$ (San Pablo de Tregua, $39^{\circ} 35^{\prime} \mathrm{S}-72^{\circ} 05^{\prime} \mathrm{O}$ ), en una ladera de exposición sur anteriormente cubierta por Chusquea culeou E. Desv. que floreció los años 2000 y 2001 y luego murió, como es normal para ésta y otras bambúceas (González et al. 2002). La vegetación arbórea remanente estaba formada por algunos individuos aislados de Saxegothaea conspicua Lindl., Nothofagus dombeyi (Mirb.) Oerst. y Laureliopsis philippiana (Looser) Schodde, especies comunes del tipo forestal Coihue-Raulí-Tepa propio de la zona, pero carecía de $N$. nervosa, otra de las especies comunes de este tipo forestal (Donoso 1981).

El clima del sector de estudio, según Koeppen, es oceánico costero con suave influencia mediterránea, con veranos cortos y secos e inviernos muy húmedos. La temperatura media anual es de $11^{\circ} \mathrm{C}$, la temperatura media del mes más frío de $5^{\circ} \mathrm{C}$ (agosto) y la del mes más cálido de $20^{\circ}$ $\mathrm{C}$ (febrero). El número de heladas anuales varía entre 30 y 50 días, concentrándose de agosto a septiembre (Neira 2005). El suelo pertenece a la serie Liquiñe (Acrudoxic hapludand) y es derivado de cenizas volcánicas modernas, estratificadas, sobre un horizonte de pumicita pronunciado que yace sobre rocas andesíticas-basálticas (CIREN 1999). Otras características del suelo en el área de estudio han sido descritas por Donoso et al. (2007a).

Las plantas utilizadas fueron originadas de semillas de la misma procedencia donde se instaló el ensayo y producidas en una temporada (período 2003-2004) en contenedores de $140 \mathrm{~cm}^{3}$, en el vivero Bosques del Sur de la Universidad Austral de Chile, en Valdivia. Se utilizó sustrato de corteza compostada de Pinus radiata D. Don, el cual fue mezclado con tres dosis de FLC recubierto con polímero [Osmocote ${ }^{\circledR}$ (15-9-12)]: 2,5, 5,0 y 7,5 $\mathrm{kg} \mathrm{m}^{-3}$. Como las plantas no se pueden producir en contenedores con sustrato inerte sin la adición de nutrientes, se considera que la dosis menor correspondió al control del ensayo. La liberación de estos elementos, según el fabricante ${ }^{1}$, puede ser de 5-6 meses a

\footnotetext{
Scotts-Sierra, Horticultural Products Company, 14111 Scottslawn Rd., Marysville, OH, United States.
} 
temperatura de $32^{\circ} \mathrm{C}$, de $8-9$ meses a temperatura de $27^{\circ}$ $\mathrm{C}$ y de $9-10$ meses a temperatura de $21^{\circ} \mathrm{C}$.

Al finalizar el período de producción en vivero las plantas presentaron mayor desarrollo con ambas dosis altas de FLC y mostraron diferencias significativas (ANDEVA, $P<0,05)$ en las medias de altura, diámetro de cuello y peso seco total (Bustos et al. 2008). La altura de la planta y su diámetro de cuello han sido tradicionalmente identificadas como variables predictoras importantes del desarrollo de plantas una vez establecidas en plantaciones en terreno (Wilson y Jacobs 2006, Navarro et al. 2006). Las plantas con altura mayor a $35 \mathrm{~cm}$ y diámetro de cuello mayor a $3 \mathrm{~mm}$ fueron podadas para homogeneizar su altura entre 35 y $40 \mathrm{~cm}$, es decir, aproximadamente el doble del largo de la raíz $(16 \mathrm{~cm})$ logrado con el tipo de contenedor usado. Estas plantas fueron podadas a principios de marzo en vivero, cuando su altura ya está bastante definida. Con esto se pretendió reducir la relación altura/diámetro de cuello, de modo de obtener teóricamente un mejor balance entre la raíz y la parte aérea. Como las plantas con dosis más baja de FLC tuvieron una altura media de $24,3 \mathrm{~cm}$, el tratamiento con poda de éstas se llevó a cabo en aquellas más altas de esta población.

La plantación se realizó durante julio del 2004. Previamente, se procedió a rozar de manera manual la superficie cubierta por densa cobertura de Ch. culeou, después se arrumaron los desechos en fajas de ancho promedio de $2 \mathrm{~m}$, con $4 \mathrm{~m}$ intermedios libres de desechos para la plantación. Cada uno de los seis tratamientos (tres dosis de FLC con y sin poda apical) tuvo tres repeticiones. Cada parcela (repetición) fue rectangular, de 15 plantas. Aunque no hubo diferencias de tamaño entre las plantas producidas con 5,0 y $7,5 \mathrm{~kg} \mathrm{~m}^{-3}$ de FLC, se mantuvieron las tres dosis de fertilizantes como niveles del factor FLC en la evaluación de la plantación debido a que podrían presentar diferentes niveles de reserva nutritiva en sus tejidos. Esto último no fue evaluado en las plantas del vivero.

La supervivencia se evaluó con un análisis de varianza no paramétrico de Kruskal-Wallis (H-test), para el factor dosis de FLC, y la prueba de Mann-Whitney (U-test) para el factor poda apical, utilizando las parcelas como unidad muestral. La supervivencia fue evaluada en el mes de mayo de cada año, es decir, durante el período en que $N$. nervosa no tiene hojas (abril-septiembre en esta región). Los incrementos anuales en altura (primer, segundo y tercer año de evaluación) fueron sometidos a un análisis de covarianza y se empleó como covariable la altura inicial de plantación (2004), usando como unidad muestral cada planta. El diámetro a la altura del pecho (DAP) al año tres también se sometió a un análisis de varianza con cada planta como unidad muestral. Adicionalmente, las plantas de las dos dosis más altas de FLC, con y sin poda apical, fueron evaluadas mediante un análisis de varianza factorial de 2 × 2 , de modo de indagar la probable inte- racción de los factores FLC y poda apical en el desarrollo de las plantas. No se incluyó en este análisis factorial al conjunto de plantas que recibió la menor dosis de FLC, debido a que aquellas con poda apical no representaban la media de ese tratamiento de fertilización en vivero, sino que correspondieron a una muestra de plantas de mayor crecimiento en altura que permitió aplicarles poda apical. Para todos los análisis se consideró una probabilidad de $5 \%(P<0,05)$ (Sokal y Rohlf 1995). La normalidad de los datos se constató por medio de la prueba Kolmogorov y Smirnov, y la homocedasticidad mediante la prueba de Levene. Los análisis estadísticos se realizaron mediante el programa estadístico SPSS 11.5.

\section{RESULTADOS}

La supervivencia no fue afectada significativamente $(P>$ $0,05)$ por la dosis de FLC ni por la poda apical en ninguno de los tres años evaluados (figura 1). La interacción de estos dos factores tampoco fue significativa. Sin embargo, las tendencias medias de la supervivencia fueron consistentes en los tres años observados, con mayores valores al incrementar la dosis de fertilizante y en las plantas con poda apical. A través del tiempo la supervivencia general en el ensayo decreció desde $82 \%$ el primer año a $70 \%$ en el segundo y a $62 \%$ en el tercero. En todos los casos se observó alta variabilidad en los porcentajes de supervivencia, los cuales estarían asociados a heladas tardías de primavera y algunas en verano (Soto et al. 2009).

Los incrementos anuales en altura y DAP tampoco fueron afectados significativamente $(P>0,05)$ por la FLC ni por la poda apical en los tres años de evaluación de la plantación (cuadro 1 y figura 2). Esto significó que en las plantas sin poda apical la diferencia inicial en la altura se mantuviera en el tiempo (cuadro 1). En las plantas con poda, que comenzaron con una altura similar entre ellas (cuadro 1), no hubo diferencias significativas en crecimiento entre dosis, aunque aquellas con la dosis de $5 \mathrm{~kg} \mathrm{~m}^{-3}$ de FLC mostraron una tendencia de mayor crecimiento acumulado al año 3 . La poda apical produjo sólo una tendencia de mayor incremento en altura con respecto a las plantas sin poda apical (cuadro 1). Las alturas finales promedio al tercer año fluctuaron entre $143,6 \pm 52,4 \mathrm{~cm}$ y $174,4 \pm 54,4 \mathrm{~cm}$ (cuadro 1 ). En general, el ensayo no mostró una incidencia temporal en el crecimiento en altura (años 1, 2 y 3), tanto para las dosis de FLC, la poda apical y la interacción entre los tratamientos (cuadro 1).

Los resultados arrojaron que tampoco hubo interacción significativa entre la fertilización y la poda apical para los dos tratamientos de mayor dosis de FLC en la variable incremento en altura, en ninguno de los tres años de evaluación $(P=0,968$ al año $1 ; P=0,304$ al año 2 , y $P=0,263$ al año 3). 

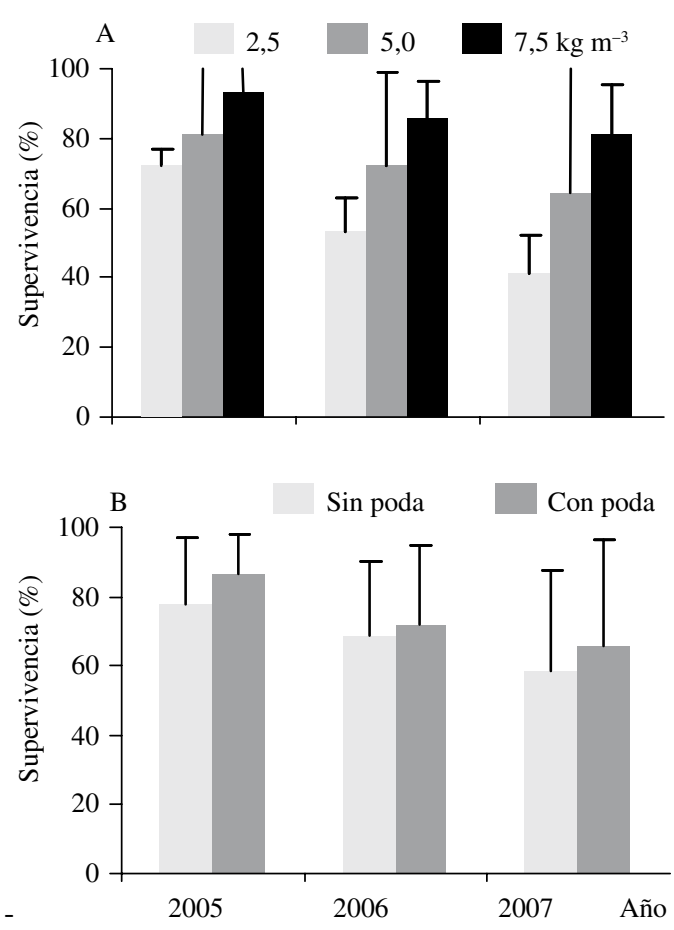

Figura 1. Supervivencia promedio según dosis de FLC (A) y poda apical (B) en los tres años evaluados (líneas verticales representan la desviación estándar).

Average survival according to doses of FLC (Osmocote) (A) and topping (B) during the three years of evaluation (vertical lines represent standard deviation).

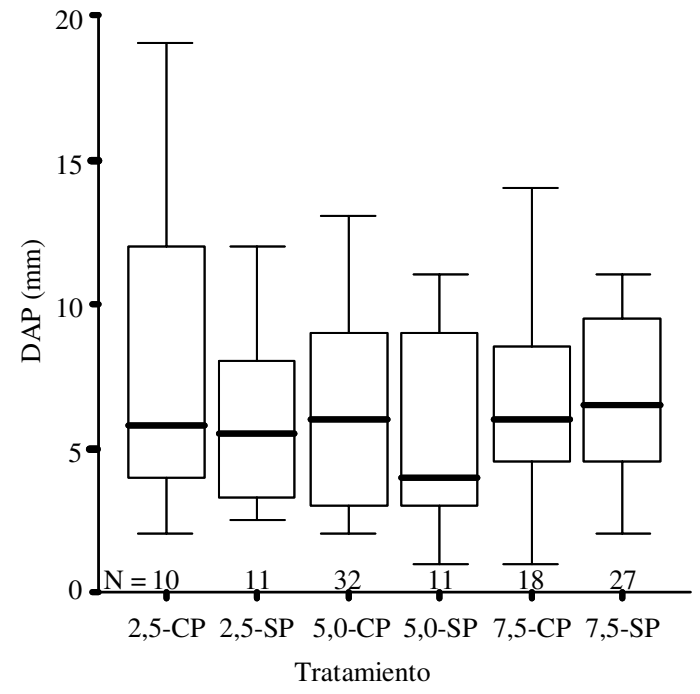

Figura 2. Distribución del diámetro del tallo a la altura del pecho (DAP) luego del tercer año de crecimiento de la plantación. Líneas centrales representan la mediana; valores extremos de la caja representan el primer y tercer cuartil; en el eje X: cifras indican la dosis de fertilizante y letras la condición de poda $(\mathrm{CP}=$ con poda; $\mathrm{SP}=\sin$ poda) $\mathrm{N}=$ número de plantas.

Boxplot representation of the DBH distribution at the third year of plantation. The central line shows the median; extreme values of the box represent the first and third quartiles. In the $\mathrm{X}$-axis numbers indicate the dose of fertilizer, and letters the condition of pruning $(\mathrm{CP}=$ with pruning; $\mathrm{SP}=$ without pruning); $\mathrm{N}=$ number of plants.

Cuadro 1. Crecimiento acumulado en altura (cm) según dosis de FLC para plantas con y sin poda apical. Cumulative height growth according to FLC (Osmocote) doses for plants with and without top pruning.

\begin{tabular}{lcccc}
\hline Poda & Dosis FLC $\left(\mathrm{kg} \mathrm{m}^{-3}\right)$ & 2005 & 2006 & 2007 \\
\hline \multirow{3}{*}{ Con poda } & 2,5 & $67,3 \pm 20,7$ & $125,2 \pm 38,9$ & $163,3 \pm 60,5$ \\
& 5,0 & $65,2 \pm 16,7$ & $126,4 \pm 32,1$ & $174,4 \pm 54,4$ \\
& 7,5 & $63,3 \pm 13,0$ & $118,7 \pm 34,9$ & $144,9 \pm 52,4$ \\
\hline \multirow{3}{*}{ Sin poda } & 2,5 & $60,2 \pm 18,6$ & $96,7 \pm 34,2$ & $143,6 \pm 52,4$ \\
& 5,0 & $70,7 \pm 11,5$ & $119,1 \pm 25,0$ & $156,5 \pm 40,9$ \\
& 7,5 & $68,4 \pm 17,0$ & $115,5 \pm 30,9$ & $161,6 \pm 46,8$ \\
\hline & Valor F poda & 2,$206 ; P=0,140$ & 1,$022 ; P=0,314$ & 0,$347 ; P=0,557$ \\
& Valor F dosis & 1,$471 ; P=0,227$ & 0,$274 ; P=0,602$ & 1,$459 ; P=0,229$ \\
& Valor F interacción & 0,$002 ; P=0,968$ & 1,$066 ; P=0,304$ & 1,$266 ; P=0,263$ \\
\hline
\end{tabular}

\section{DISCUSIÓN Y CONCLUSIONES}

En los tres años de evaluación en terreno $N$. nervosa no respondió de manera significativa a los tratamientos culturales aplicados en vivero, tanto en supervivencia (figura 1) como en crecimiento en altura (cuadro 1) y diámetro (figura 2), no obstante que las plantas establecidas en la plantación habían sido afectadas en su desarrollo por la aplicación de FLC en el vivero (Bustos et al. 2008). Si la calidad de una planta obtenida en el vivero se evalúa de acuerdo a su desempeño en terreno, como corresponde (Wilson y Jacobs 2006, Navarro et al. 2006), los datos obtenidos no otorgan evidencia suficiente para clasificar las plantas de vivero en distintas categorías de calidad según la práctica de cultivo que se les aplicó. Los resultados no evidencian mejores crecimientos en cuanto al 
incremento con plantas que tuvieron mayores dosis de fertilización en vivero, pero sí sugieren que es posible lograr una mayor supervivencia con ese tipo de plantas (dosis media y alta de FLC) que llegan a terreno con una mayor biomasa. Ello es consistente con los resultados obtenidos por Grosse (1988), también en plantaciones de $N$. nervosa a campo abierto (supervivencia de $35 \%$ en plantas de no fertilizadas y de $80 \%$ con plantas fertilizadas en la Cordillera de Malleco ( $\left.38^{\circ} \mathrm{S}\right)$ ). Los resultados de supervivencia en el presente estudio sugieren que puede ser conveniente generar plantas con dosis de 5 a $7,5 \mathrm{~kg} \mathrm{~m}^{3}$ de fertilización, ya que el costo de plantación es siempre alto y la pérdida de muchas plantas debido a la mortalidad inicial puede ser un gran detrimento en la inversión. En terreno hubo en promedio un $25 \%$ menos de supervivencia de plantas con la menor dosis de fertilización con respecto a aquellas de mayor dosis en el primer año, y esta diferencia aumentó aproximadamente a un $50 \%$ terminado el tercer año (figura 1 ).

Las plantas con mayores dosis de fertilización no sometidas a poda apical comenzaron con mayor altura que aquellas con menor dosis de fertilización, y esa diferencia la mantuvieron de modo más o menos constante durante los tres años evaluados. Es decir, no tuvieron un incremento superior que aquellas con menor fertilización. Entre las plantas con poda apical, que comenzaron con alturas similares, tampoco hubo diferencias en incremento. Las plantas con poda apical sólo mostraron una tendencia de mayor supervivencia que aquellas sin poda apical (en promedio $<10 \%$ ), lo cual puede obedecer a que la masa eliminada con la poda aérea para homogeneizar la altura en $35-40 \mathrm{~cm}$ fue de pequeña cuantía.

Estos resultados no muestran una correspondencia con la literatura del tema, la que señala la existencia de efectos residuales en terreno de la FLC (Oliet et al. 1999, Rose et al. 2004, Jacobs et al. 2005, Hathaway y Whitcomb 1977) y que las plantas con mayor diámetro de cuello o menor relación H/D (lo que se obtiene con poda apical) logran mejores desempeños en terreno, al menos en zonas templadas (Wilson y Jacobs 2006). Son varias las posibles respuestas a la ausencia de efectos significativos de estas actividades en el desarrollo en terreno de las plantas. En primer lugar, es posible que el efecto residual del FLC no haya existido o haya sido marginal, ya que su aplicación original fue hecha para nutrir las plantas durante su desarrollo en vivero (el fertilizante aplicado tendría un efecto de 10 meses a $21^{\circ} \mathrm{C}$, es decir, de más tiempo -no definido ni etiquetado en el producto- con una temperatura media de $11^{\circ} \mathrm{C}$ en terreno). En segundo lugar, si bien se ha establecido que, en general, hay una relación positiva entre el mayor éxito en la plantación y el diámetro de cuello de las plantas (que fue mayor en plantas con mayor FLC, según Bustos et al. (2008)), el rango de diámetros tal vez fue estrecho para que luego se manifestaran diferencias en terreno. En cuanto a la poda apical, esta no tuvo efecto probablemente debido a que la masa extraída a las plantas fue marginal, como se señalara anteriormente. Los resultados sugieren que la poda apical no tuvo efectos significativos, pero que las dosis mayores (5 y $7,5 \mathrm{~kg} \mathrm{~m}^{3}$ ) de FLC tenderían a generar una mayor supervivencia de las plantas. El diseño experimental usado no permite señalar con certeza si este efecto fue debido al hecho de usar plantas más grandes y con mejores reservas nutritivas o si, además de ello, se debió a la liberación de nutrientes residuales del fertilizante encapsulado. De todas formas, los resultados demuestran que con el rango de calidad de planta obtenida en vivero se pueden alcanzar similares resultados en terreno.

\section{AGRADECIMIENTOS}

Al proyecto FONDEF D04I1271 y al convenio CONAF-UACh. También a los colegas Bernardo Escobar y Daniel Uteau por sus comentarios a versiones iniciales del manuscrito.

\section{REFERENCIAS}

Alzugaray P, D Haase, R Rose. 2004. Efecto del volumen radicular y la tasa de fertilización sobre el comportamiento en terreno de plantas de pino oregón (Pseudotsuga menziesii (Mirb.) Franco) producidas con el método 1+1. Bosque 25(2): 17-33.

Bustos F, ME González, PJ Donoso, V Gerding, C Donoso, B Escobar. 2008. Efectos de distintas dosis de fertilizante de entrega lenta (Osmocote) en el desarrollo de plantas en contenedor de Nothofagus dombeyi, Nothofagus nervosa y Eucryphia cordifolia. Bosque 28(2): 155-161.

CIREN (Centro de Información de Recursos Naturales, CL). 1999. Estudio agrológico de la provincia de Valdivia, X Región. Descripciones de suelos. Materiales y símbolos. Santiago, Chile. CIREN. 199 p.

Day DC, JM Kabrick, M Gold. 2006. The role of large container seedlings in afforesting oaks in bottomlands. In Connor KF ed. Proceedings of the $13^{\text {th }}$ biennial southern silviculture research conference. Gen. Tech. Rep. SRS-92. Ashville, NC: US Dept. Agriculture, Forest Service, Southern Research Station. 640 p.

Díaz-Vaz JE, H Poblete, R Juacida, F Devliger. 2002. Maderas comerciales de Chile. Valdivia, Chile. Marisa Cuneo Ediciones. 91 p.

Donoso C. 1981. Tipos forestales de los bosques nativos de Chile. Documento de trabajo $\mathrm{N}^{\mathrm{o}} 38$ Investigación y Desarrollo Forestal (CONAF/PNUD/FAO). Santiago, Chile. Publicación FAO. $82 \mathrm{p}$.

Donoso PJ, ME González, B Escobar, I Basso, L Otero. 1999. Viverización y plantaciones de raulí, roble y coihue. In Donoso C y A Lara eds. Silvicultura de los bosques nativos de Chile. Santiago, Chile. Universitaria. p. 177-244.

Donoso PJ, V Gerding, D Uteau, DP Soto, O Thiers, C Donoso. 2007a. Crecimiento y mortalidad inicial de una plantación de Nothofagus dombeyi con diferentes dosis de fertilizantes en la Cordillera de Los Andes. Bosque 27(3): 249-255. 
Donoso PJ, DP Soto, C Donoso, H Rivera. 2007b. Crecimiento inicial en altura para plantaciones de coihue, roble y raulí en altitudes medias en la provincia de Valdivia, Chile. Bosque Nativo 40: 8-12.

Duryea ML, TD Landis. 1984. Forest nursery manual: production of bareroot seedlings. The Hage, The Netherlands. Martinus Nijhoff/Junk Publishers. 386 p.

Duryea ML, P Dougherty. 1991. Forest regeneration manual. The Hage, The Netherlands. Kluwer Academic Publishing. $233 \mathrm{p}$.

Fan Z, JA Moore, DL Wenny. 2004. Growth and nutrition of container-grown ponderosa pine seedlings with controlledrelease fertilizer incorporate in the root plug. Annals of Forest Science 61: 117-124.

González ME, TT Veblen, C Donoso, L Valeria. 2002. Tree regeneration responses in a lowland Nothofagus- dominated forest after bamboo dieback in South Central Chile. Plant Ecology 161: 59-73.

Grosse H. 1988. Crecimiento de plantaciones con raulí y roble bajo dosel en dependencia del grado de luminosidad y fertilización. Ciencia e Investigación Forestal (2): 13-29.

Hall M, J Witte. 1998. Maderas del sur de Chile. Árboles, aplicaciones y procesos. Santiago, Chile. IER edicionesDED. $91 \mathrm{p}$.

Hathaway RD, CE Whitcomb. 1977. Propagation of Quercus seedlings in bottomless containers with osmocote. Journal of Arboriculture 3(11): 208-212.

Holmes PM, DM Richardson. 1999. Protocols for restoration based on recruitment dynamics, community structure, and ecosystems function. Restoration Ecology 7(3): 215-230.

Jacobs DF, KF Salifu, JR Seifert. 2005. Growth and nutritional response of hardwood seedlings to controlled-release fer- tilization at outplanting. Forest Ecology and Management 214: 28-39.

Navarro RM, P Villar-Salvador, A del Campo. 2006. Morfología y establecimiento de plantones. In Cortina J, JL Peñuelas, J Puértolas, R Savé, A Vilagrosa eds. Calidad de planta forestal para la restauración en ambientes mediterráneos. Estado actual de conocimientos. Madrid, España. Ministerio de Medio Ambiente, Serie Forestal. p. 67-88.

Neira E. 2005. Producción de agua en dos microcuencas con diferentes coberturas de renovales de Nothofagus en la Cordillera de Los Andes (San Pablo de Tregua). Tesis de Magíster en Ciencias. Valdivia, Chile. Facultad de Ciencias Forestales, Universidad Austral de Chile. 84 p.

Oliet J, ML Segura, FM Domínguez, E Blanco, R Serrada, M López, F Artero. 1999. Fertilizantes de liberación controlada lenta aplicados a la producción de plantas forestales de vivero. Efecto de dosis y formulación sobre la calidad de Pinus halepensis. Investigación Agraria: Sistemas y Recursos Forestales 8(1): 207-228.

Otero L. 2006. La Huella del Fuego: Historia de los bosques nativos, poblamiento y cambios en el paisaje en el sur de Chile. Santiago, Chile. Pehuén. 172 p.

Rose R, DL Haase, E Arellano. 2004. Fertilizantes de entrega controlada: potencial para mejorar la productividad de la reforestación. Bosque 25(2): 89-100.

Sokal R, F Rohlf 1995. Biometry. San Francisco, USA. Freeman and Company. $887 \mathrm{p}$.

Soto DP, PJ Donoso, D Uteau, A Zúñiga-Feest. 2009. Environmental factors affect the spatial arrangement of survival and damage of outplanted Nothofagus dombeyi seedlings in the Chilean Andes. Interciencia 34(2): 100-105.

Wilson BC, DF Jacobs. 2006. Quality assessment of temperate zone deciduous hardwood seedlings. New Forests 31:417-433.

Recibido: 09.07.08

Aceptado: 12.01.09 